\section{G-CSF-Gabe bei Chemotherapie}

\section{Weniger Neutropenien mit Lipegfilgrastim}

Langwirksame G-CSF-Präparate erleichtern sowohl dem Patienten als auch dem Arzt die Reduktion der Inzidenz der Chemotherapiebedingten febrilen Neutropenie, da sie nur 1-mal pro Zyklus injiziert werden müssen. Mit Lipegfilgrastim (Lonquex ${ }^{\circledR}$ ) gibt es seit kurzem eine neue Option, die sich als mindestens so effektiv wie Pegfilgrastim erwiesen hat".

Das Auftreten einer febrilen Neutropenie (FN) bringt 2 wesentliche Probleme mit sich. Zum einen erhöht die FN die Mortalität der Patienten [1]. Zum anderen zwingt sie dazu, die Intensität der weiteren Chemotherapie zu verringern. Dies geht auf Kosten ihrer Wirksamkeit, wie Prof. Dr. med. Hartmut Link, Kaiserslautern, ausführte [2]. Um einer FN vorzubeugen, empfiehlt die EORTC-Leitlinie [3] eine primäre G-CSFGabe, wenn das FN-Risiko einer Chemotherapie $20 \%$ übersteigt. Liegt es unter $10 \%$, ist G-CSF verzichtbar. Bei einem Risiko zwischen 10 und $<20 \%$ geben Patienten-bezogene Risikofaktoren den Ausschlag.

\section{Neue Pegylierung}

Langwirksame Präparate machen die G-CSFGabe besonders einfach. Die Halbwertszeit des kurzwirksamen Filgrastim, welches täglich appliziert werden muss, konnte durch Molekülmodifikationen erheblich verlängert werden. Während das erste langwirksame rekombinante
G-CSF Pegfilgrastim ein chemisch N-terminal pegyliertes Molekül ist [4], ging man für Lipegfilgrastim einen neuen Weg, erklärte Prof. Dr. rer. nat. Theodor Dingermann, Frankfurt. Hier wurde das Molekül enzymatisch an der Position Threonin 134 pegyliert, der Stelle, an die auch im natürlichen G-CSF eine Zuckerstruktur gebunden ist [5].

Die Pegylierung vergrößert die 6 Moleküle, sodass sie nicht über die Nieren ausgeschieden, sondern nur durch die Neutrophilen-Elastase abgebaut werden können. Eine In-vitro-Studie hat gezeigt, dass Lipegfilgrastim gegen diesen Abbau stabiler ist als Pegfilgrastim [6].

\section{Nicht-Unterlegenheit belegt}

Eine multizentrische, randomisierte Phase-IIIStudie [7] konnte belegen, dass Lipegfilgrastim dem Pegfilgrastim in seiner klinischen Wirkung nicht unterlegen ist. Die Dauer der schweren Neutropenie (Grad 4, ANC $\left.<0,5 \times 10^{9} / \mathrm{l}\right)$ im ersten Zyklus (primärer Endpunkt) betrug 0,7 \pm 0,9 Tage in der Lipegfilgrastim-Gruppe und $0,8 \pm 0,9$ Tage in der Pegfilgrastim-Gruppe ( $p=$ 0,1260). In einigen sekundären Endpunkten nicht alle waren signifikant - waren Patienten der Lipegfilgrastim-Gruppe etwas im Vorteil, z.B. in der Erholungszeit der Neutrophilen, in der Inzidenz der schweren Neutropenie und in der Auswirkung auf die weitere Chemotherapie. Bei keiner Patientin, die Lipegfilgrastim erhielt, musste in den Zyklen 2-4 eine Dosis ausgelassen oder reduziert werden, während dies bei 8 Patientinnen der Pegfilgrastim-Gruppe der Fall war, betonte Prof. Dr. med. Helmut Ostermann, München. Die Dosisreduktionen und Zyklusverschiebungen können auf verschiedenen Ursachen beruhen.

An der Studie hatten 202 Patientinnen mit Brustkrebs im Stadium II-IV teilgenommen, die eine Chemotherapie mit maximal 4 Zyklen Docetaxel und Doxorubicin bekamen. Jeweils am 2. Tag eines jeden Zyklus wurden $6 \mathrm{mg}$ Lipegfilgrastim oder $6 \mathrm{mg}$ Pegfilgrastim subkutan gespritzt.

\section{Dr. Angelika Bischoff, Planegg}

*ZZur Verkürzung der Dauer von Neutropenien und zur Verminderung der Inzidenz von febriler Neutropenie bei erwachsenen Patienten, die wegen einer malignen Erkrankung eine zytotoxische Chemotherapie erhalten (mit Ausnahme von chronisch-myeloischer Leukämie und myelodysplastischen Syndromen)

\section{Quelle}

31. Deutscher Krebskongress, Berlin, Symposium «G-CSF optimieren = Therapie optimieren», 20. Februar 2014

\section{Referenzen}

1 Kuderer NM et al.: Cancer 2006;106:2258-2266.

2 Lyman GH et al.: Ann Oncol 2013;24: 2475-2484.

3 Aapro MS et al.: Eur J Cancer 2011;47:8-32.

4 Fachinformation Neulasta, Stand 11/2013.

5 Fachinformation Lonquex, Stand 7/2013.

6 EPAR Assessment report Lonquex EMA/371234/2013. www.ema.europa.eu/docs/en_GB/document_library/ EPAR_-_Public_assessment_report/human/002556/ WC500148382.pdf.

7 Bondarenko I et al.: BMC Cancer 2013;13:386-397.

Primäre G-CSF-Prophylaxe nach Leitlinien [3]: Neue internetbasierte Entscheidungshilfe Häufig ist es schwierig, Angaben zum FN-Risiko einer bestimmten Chemotherapie aufzufinden. Eine Internet-basierte Datenbank mit modular zusammengesetzten Therapieprotokollen in der Onkologie (www.onkopti.de) wird dies in Zukunft erleichtern. Hier findet der Arzt alle relevanten Daten zu verschiedenen gebräuchlichen Chemotherapien in übersichtlichter Form, unter anderem zur Höhe des Neutropenie-Risikos und zur empfohlenen G-CSF-Prophylaxe. Die Datenbank soll ein leitliniengerechtes Vorgehen z.B. in der G-CSF-Prophylaxe erleichtern, wie Prof. Link betonte.
Weitere Informationen bei

Teva GmbH

Ulrike Krieger-Ballhausen

Graf-Arco-Straße 3, 89079 Ulm

ukrieger-ballhausen@teva.de

\title{
PharmaTicker+++ PharmaTicker+++ PharmaTicker+++ PharmaTicker+++
}

Lilly. Die internationale Phase-III-Studie REVEL zur Zweitlinientherapie des nichtkleinzelligen Lungenkarzinoms (NSCLC) hat ihren primären Endpunkt erreicht. In der Studie konnte mit einer Kombinationstherapie aus dem Angiogenesehemmer Ramucirumab (IMC-1121B) plus Docetaxel eine Verlängerung des Gesamtüberlebens bei NSCLC-Patienten mit plattenepithelialer und nichtplattenepithelialer Histologie im Vergleich zu Docetaxel plus Placebo erzielt werden.

3K Agentur für Kommunikation $\mathrm{GmbH}$ Elisabeth Fauth

elisabeth.fauth@3k-kommunikation.de
Astellas. Der Gemeinsame Bundesausschuss (G-BA) hat einen beträchtlichen Zusatznutzen von Enzalutamid (XTANDI ${ }^{\mathrm{TM}}$ ) zur Behandlung von Patienten mit metastasiertem kastrationsresistentem Prostatakarzinom nach einer vorherigen Docetaxel-basierten Chemotherapie zugesprochen. Grundlage für die Entscheidung sind die Daten, die in der Zulassungsstudie AFFIRM gewonnen wurden. In dieser Studie zeigte sich ein statistisch signifikant verlängertes Gesamtüberleben durch Enzalutamid gegenüber Placebo.

Astellas Pharma GmbH

Dr. Marco Groß-Langenhoff

marco.gross-langenhoff@astellas.com
Hexal. Ab dem 1. Juni, 7 Uhr bietet der Online-Service ASCO DIRECT ${ }^{\mathrm{TM}}$ wieder kompakte News vom 2014 ASCO $^{\circledR}$ Annual Meeting. Auf www.asco-direct.de gibt es die neuen, relevanten Studienergebnisse zu verschiedenen Tumorentitäten. Neu in diesem Jahr ist die optimierte Anwendungsstruktur, die eine Vollbildanzeige der Abstracts ermöglicht. Hexal präsentiert ASCO DIRECT ${ }^{\mathrm{TM}}$ von Anfang an exklusiv für Deutschland. Dieser Informationsservice ist von der ASCO $^{\circledR}$ offiziell lizenziert.

www.asco-direct.de
KARGER

Fax +497614520714 Information@Karger.con 


\section{Exjade $^{\circledR}$ (Deferasirox):}

\section{Eisen in Balance - Therapiemanagement bei MDS- und Thalassämie-Patienten}

Im Rahmen des 31. Deutschen Krebskongresses fand das Novartis Oncology Meet the Experts "Eisen in Balance: Therapiemanagement bei MDS- und Thalassämie-Patienten» statt. Referenten waren Prof. Dr. Wolf-Karsten Hofmann, Direktor der III. Medizinischen Klinik der Universitätsmedizin Mannheim, der zu Myelodysplastischen Syndromen (MDS) referierte, und Dr. Regine Grosse, Ärztin an der II. Medizinischen Klinik und der Kinder-Hämatologie und-Onkologie des Universitätsklinikums Hamburg-Eppendorf, die sich den Thalassämien widmete.

\section{Eisenüberladung bei MDS}

MDS sind erworbene Erkrankungen des Knochenmarks bzw. des Blutes, die durch eine ineffektive Blutbildung im Knochenmark gekennzeichnet sind [1]. Von den Reifungs- und Funktionsstörungen können eine oder mehrere Zelltypen im Blut betroffen sein [1]. MDS können zudem bei manchen Patienten zu einer akuten myeloischen Leukämie (AML) führen [1]. Ein großer Teil der MDS-Patienten entwickelt im Laufe der Erkrankung eine Anämie und ist dann auf eine regelmäßige Gabe von Erythrozytenkonzentrat(EK)Einheiten angewiesen. Täglich verliert der Körper 1-2 mg Eisen über Darm, Urin und Haut [2]. Eine EK-Einheit enthält jedoch $250 \mathrm{mg}$ Eisen, sodass es durch die kontinuierliche Zufuhr von Eisen über die Transfusionen bei chronisch transfundierten MDS-Patienten zu einer Eisenüberladung z.B. in Leber und im Herzen kommt [3]. Diese Anreicherung von Eisen ist mit Hilfe eines Eisenchelators wie Deferasirox behandelbar [1].

Von einer Therapie mit Deferasirox profitieren chronisch transfundierte MDS-Patienten in vielerlei Hinsicht: Die Hämatopoese kann sich verbessern [4-6] und der Transfusionsbedarf als Folge davon sinken [6]. Eine bei der jüngsten
Jahrestagung der American Society of Hematology (ASH) in New Orleans vorgestellte US-amerikanische Registerstudie zeigt außerdem, dass MDS-Patienten, die mindestens 6 Monate lang eine Eisenchelat-Therapie erhalten hatten, mehr als doppelt so lang lebten (median 102,5 vs. 48,7 Monate; $p<0,0001$ ) und später und seltener eine AML entwickelten (nach median 77 vs. 45,6 Monaten; $p<0,0001$ ) als Betroffene ohne Eisenchelation [7].

Deferasirox reduziert Eisenüberladung bei $\beta$-Thalassämie major

Die Thalassämien zählen zu den Hämoglobinopathien. Darunter versteht man eine Gruppe von angeborenen hämatologischen Erkrankungen, die auf eine Störung der Hämoglobin-Produktion, verursacht durch Mutationen in den Hämoglobin-Genen, zurückzuführen sind [8]. Abhängig davon, von welchem Thalassämie-Syndrom der Patient betroffen ist, kommt es zu einer mehr oder weniger ineffektiven Hämatopoese. Die $\beta$-Thalassämie major ist die in der Regel homozygote oder gemischt-heterozygote $\beta$-Thalassämie-Form [8]. Je stärker die Hämatopoese gestört ist, desto eher benötigen die Patienten regelmäßige Bluttransfusionen. Da es sich um eine Erbkrankheit handelt, die zurzeit noch nicht kurativ behandelt werden kann - außer in Einzelfällen mit Hilfe einer Stammzelltransplantation - muss die Transfusionsbehandlung bereits im ersten Lebensjahr beginnen und lebenslang erfolgen [8]. Auch hier kommt es aufgrund der chronischen Transfusionsabhängigkeit der Thalassämie-Patienten zur Entwicklung einer Eisenüberladung, die wiederum mithilfe einer Eisenchelat-Therapie mit Deferasirox behandelt werden kann. Erfolgt keine Eisenchelation, versterben die Betroffenen an den Folgen der Organschäden, die durch die
Eisenüberladung verursacht werden, meist bereits vor Erreichen des 30. Lebensjahres [9].

Die Therapie mit Deferasirox bietet auch den Thalassämie-Patienten Vorteile: Die Leber- und Herzeisenüberladung wird reduziert [10], eine Anreicherung von Eisen in den hormonbildenden Organen wird verhindert [11] - dies wirkt sich positiv auf das Überleben aus [12]. Die Lebensqualität kann sich durch die nur 1-mal tägliche orale Verabreichung verbessern. Im Vergleich zu der wesentlich weniger komfortablen subkutanen Dauerinfusion von Deferoxamin [13] zeigt Deferasirox [14] die gleiche Wirksamkeit, wie sich in der Verlängerung einer internationalen Phase-II-Vergleichsstudie zwischen Deferasirox und Deferoxamin zeigte, die ebenfalls beim ASH-Kongress vorgestellt wurde [10].

\section{Quelle}

Novartis Oncology Meet the Experts «Eisen in Balance: Therapiemanagement bei MDS- und Thalassämie-Patienten», im Rahmen des 31. Deutschen Krebskongresses, 20.02.2014 in Berlin

\section{Referenzen}

www.dgho-onkopedia.de/de/onkopedia/leitlinien/mds.

2 www.leukaemie-hilfe.de/download-informationen.html? \&no_cache $=1 \& t x \_d r b l o b \_p i l[d o w n l o a d U i d]=76$.

3 Leitch HA et al.: Hematology. Am Soc Hematol Educ Program. 2009:664-672.

4 Pennell DJ et al.: ASH 2013; abstr \#1018.

5 Giagounidis A et al.: TumorDiagn \& Ther 2012;3:29-33.

6 Angelucci E et al.: Blood 2012;118, abstr \#425.

7 Lyons RM et al: ASH 2013; abstr \#2775.

8 www.awmf.org/uploads/tx_szleitlinien/025-017l_S1_ Thalassaemie.pdf

9 Borgna-Pignatti C et al.: Haematologica 2004;89:1187-1193. 10 Pennell DJ et al.: ASH 2013; abstr \#1018.

11 Grosse R: Kinder- und Jugendmedizin 2013;13:A1-A8.

12 Brittenham GM: N Eng J Med 2011;364:146-156.

13 Desferal $^{\circledR}$ (Deferoxamin) Fachinformation (Stand: Mai 2011).

14 Exjade $^{\circledR}$ (Deferasirox) Fachinformation (Stand: Oktober 2013).

Weitere Informationen bei

Novartis Pharma GmbH

Ina Meyer

Roonstraße 25, 90429 Nürnberg

ina.meyer@novartis.com

\section{PharmaTicker+++ PharmaTicker+++ PharmaTicker+++ PharmaTicker+++}

LEO Pharma. Einschränkungen der Nierenfunktion bei Patienten mit venösen Thromboembolien werden bei der initialen Antikoagulation häufig nicht ausreichend berücksichtigt. Dies zeigen die Ergebnisse einer aktuellen Versorgungsstudie, die im Rahmen eines Symposiums auf der 58. Jahrestagung der Gesellschaft für Thromboseund Hämostaseforschung in Wien vorgestellt wurde. In diesem Zusammenhang wurde auch das Thrombosemanagement bei Tumorerkrankungen und bei Patienten in fortgeschrittenem Lebensalter diskutiert.

LEO Pharma GmbH

Thomas Schick

thomas.schick@leo-pharma.com
Sanofi. Seit 1 Jahr ist das Fusionsprotein Aflibercept in Kombination mit FOLFIRI für die Therapie von Patienten mit metastasiertem kolorektalem Karzinom, die bereits mit Oxaliplatin vorbehandelt sind, zugelassen. Das gilt unabhängig vom ras-Status des Tumors. Im klinischen Alltag hat sich die hohe Wirksamkeit der zusätzlichen Aflibercept-Gabe bestätigt. Darüber hinaus zeigen die Daten aus dem Praxisalltag, dass sich die Kombination gut handhaben lässt und Aflibercept in Kombination mit FOLFIRI von den Patienten gut vertragen wird.

Sanofi-Aventis Deutschland GmbH Cristina lannazzo presse@sanofi.com
Roche. Mit der Entwicklung und Zulassung des BRAF-Inhibitors Vemurafenib (Zelboraf ${ }^{\circledR}$ ) konnte der jahrzehntelange Stillstand in der Therapie des fortgeschrittenen malignen Melanoms überwunden werden. Die Erfahrungen aus der täglichen Praxis und die Ergebnisse der Phase-III-Zulassungsstudie BRIM-3 dokumentieren den langfristigen therapeutischen Erfolg sowie die hohen Ansprechraten und die gute Verträglichkeit von Vemurafenib.

Roche Pharma AG

Ulla Satzger

ulla.satzger@roche.com
KARGER

Fax +497614520714 Information@Karger.con Information @ Karg 
Yervoy $^{\circledR}$ (Ipilimumab) erhält in Europa die First-line-Zulassung für die Behandlung erwachsener Patienten mit fortgeschrittenem Melanom

Am 31. Oktober 2013 erhielt Yervoy ${ }^{\circledR}$ (Ipilimumab) die Zulassung der Europäischen Kommission als First-line-Therapie bei erwachsenen Patienten mit fortgeschrittenem (nichtresezierbarem oder metastasiertem) Melanom [1]. Zum Zeitpunkt der ersten Zulassung von Ipilimumab für die Therapie Erwachsener mit vorbehandeltem, fortgeschrittenem Melanom im Juli 2011 stellte die Substanz bei dieser Erkrankung den ersten größeren Behandlungsfortschritt innerhalb der letzten 30 Jahre dar, der erstmals in einer Phase-III-Studie einen Vorteil beim Gesamtüberleben (overall survival, OS) zeigte [2].

«Die First-line-Zulassung von Ipilimumab ist für die vielen Menschen in Europa, die jedes Jahr die Diagnose Melanom erhalten, von Bedeutung. Trotz Fortschritten bei First-line-Therapien des fortgeschrittenen Melanoms bestand für viele Patienten ein großer Bedarf an weiteren Behandlungsoptionen», so Han Steutel, Geschäftsführer von Bristol-Myers Squibb Deutschland. «Die Genehmigung der Zulassungserweiterung unterstreicht den Glauben an das Potenzial von Therapien wie Ipilimumab der ersten zugelassenen Substanz aus unserer immunonkologischen Pipeline. Bei BristolMyers Squibb ist die Immunonkologie ein Forschungs- und Entwicklungsbereich von hoher Priorität. Wir engagieren uns in diesem aufstrebenden und wegweisenden Therapiefeld, das die Fähigkeiten des körpereigenen Immunsystems nutzt, um Krebs zu besiegen.»

Historisch betrachtet betrug die durchschnittliche Lebenserwartung von Patienten mit metastasiertem Melanom nur 6-9 Monate [3]. «Ipilimumab ist derzeit die einzige Therapie beim fortgeschrittenen, vorbehandelten
Melanom mit nachgewiesenem anhaltendem Langzeitüberleben in einer randomisierten Phase-III-Studie [2, 4]. Mit der Zulassungserweiterung sind wir ab sofort in der Lage, unseren Patienten mit fortgeschrittenem Melanom diese innovative immunonkologische Therapie schon früher in ihrem Behandlungsverlauf anbieten zu können», erläuterte Professor Dr. med. Dirk Schadendorf, Direktor der Klinik für Dermatologie, Universitätsklinikum Essen.

Das empfohlene Induktionsregime für Ipilimumab liegt bei $3 \mathrm{mg} / \mathrm{kg}$ Körpergewicht, intravenös über einen Zeitraum von 90 min verabreicht, alle 3 Wochen für insgesamt 4 Dosen [5]. Das Nebenwirkungsprofil von Ipilimumab lässt sich weitgehend aus seinem immunstimulatorischen Wirkprinzip erklären. Für mögliche immunvermittelte Nebenwirkungen (immunrelated adverse events, irAEs) gibt es spezifische Therapiealgorithmen. Richtig und zeitnah behandelt, sind die durch Ipilimumab bedingten Nebenwirkungen in der Regel gut beherrschbar [4-6]. Immunvermittelte Nebenwirkungen können schwer oder lebensbedrohlich sein und den Gastrointestinaltrakt, die Leber, Haut, das Nerven- und Hormonsystem sowie andere Organe betreffen [4, 5]. Das Sicherheitsprofil von Ipilimumab $3 \mathrm{mg} / \mathrm{kg}$ Körpergewicht bei chemotherapienaiven Patienten aus gepoolten klinischen Phase-II/III-Studiendaten $(\mathrm{n}=75)$ und bei therapienaiven Patienten aus einer retrospektiven Beobachtungsstudie $(\mathrm{n}=120)$ war vergleichbar mit dem von Patienten mit vorbehandeltem, fortgeschrittenem Melanom [5, 7].

Die First-line-Zulassung (Zulassungserweiterung) erfolgte erstmals in der Onkologie anhand der Daten aus 2 retrospektiven Pha-
se-IV-Studien mit therapienaiven Patienten sowie anhand gepoolter Daten von Phase-IIund Phase-III-Studien mit chemotherapienaiven Patienten, die mit einer Ipilimumab-Induktionstherapie von $3 \mathrm{mg} / \mathrm{kg}$ Körpergewicht in Monotherapie behandelt wurden [1, 7, 8]. Diese retrospektiv erhobenen Daten wurden auf dem Europäischen Kongress der European Society for Medical Oncology (27.09.-01.10.2013, Amsterdam) vorgestellt.

Das Gesamtüberleben bei Ipilimumab $3 \mathrm{mg} / \mathrm{kg}$ Körpergewicht in Monotherapie bei chemotherapienaiven Patienten aus gepoolten klinischen Phase-II- und -III-Studiendaten ( $\mathrm{N}=78$; randomisiert) und bei therapienaiven Patienten aus 2 retrospektiven Beobachtungsstudien (CA184-338: $\mathrm{n}=120$ und CA184-332: $\mathrm{n}=61$ ) waren im Wesentlichen konsistent $[1,7,8]$. Die geschätzten 1-Jahresüberlebensraten lagen bei 59,5\% (95\% KI: 50,1-67,8) und 49,3\% (95\% KI: 35,6-61,6) in den beiden retrospektiven Beobachtungsstudien [1, 7,8]. Die geschätzten 1-Jahres- und 2-Jahresüberlebensraten bei chemotherapienaiven Patienten $(\mathrm{n}=78)$ aus gepoolten klinischen Phase-II- und -III-Studiendaten betrugen $54,1 \%$ (95\% KI: 42,5-65,6) bzw. $32 \%$ (95\% KI: 20,7-42,9) [1, 7, 8].

\section{Referenzen}

1 Europäische Kommission. Community register of medicinal products for human use; Stand November 2013

Eggermont A et al.: Eur J Cancer 2011;47:2150-2157.

3 Chin L et al.: Genes Dev 2006;20:2149-2182.

4 Hodi FS et al.: N Engl J Med 2010;363:711-723.

5 YERVOY $^{\circledR}$ Fachinformation; Stand Oktober 2013.

6 Robert C et al.: N Engl J Med 2011;364:2517-2526.

7 Margolin K et al.: Eur J Cancer 2013;49(2):P488.

8 Patt D et al.: Eur J Cancer 2013;49(2):P497.

\section{Stellungnahme von Bristol-Myers Squibb zur IQWiG-Bewertung für Ipilimumab bei fortgeschrittenem Melanom}

Am 17.3.2014 hat das IQWiG vor dem Hintergrund seiner methodischen Anforderungen «keinen Zusatznutzen» von Ipilimumab gegenüber der vom G-BA definierten Vergleichstherapie Dacarbazin in dem neuen Indikationsgebiet gesehen.

Wir sind jedoch davon überzeugt, dass Ipilimumab auch für therapienaive Patienten mit fortgeschrittenem Melanom eine innovative immunonkologische Therapie ist. Wir hoffen daher, dass der G-BA vor dem Hintergrund der besonderen Zulassungssituation und des großen medizinischen Bedarfs den vorgelegten adjustierten historischen Vergleich als bestverfügbare Evidenz für Yervoy ${ }^{\circledR}$ während seines Nutzenbewertungsprozesses berücksichtigt.

\section{Der G-BA Beschluss wird für Anfang Juni 2014 erwartet.}

Für das Indikationsgebiet der Patienten mit vorbehandelten fortgeschrittenem Melanom hatte der G-BA bereits im August 2012 einen «beträchtlichen» Zusatznutzen festgestellt.

Weitere Informationen bei

Bristol-Myers Squibb GmbH \& Co. KGaA

Ina Fürholzer

Arnulfstraße 29, 80636 München

Tel. +49 89 12142-304, Fax: -262

ina.fuerholzer@bms.com 